\title{
sciendo
}

\section{Biopsychosocial Effects of Competition Preparation in Natural Bodybuilders}

\author{
by \\ Andrew J. Chappell ${ }^{1}$, Trevor N. Simper ${ }^{2}$, Eric T. Trexler ${ }^{3}$, Eric R. Helms ${ }^{4}$
}

\begin{abstract}
Bodybuilders are judged on the subjective visual assessment of their muscular size, proportion, and leanness. This case series tracked four drug-free bodybuilders (3 male) during the off-season, pre-competition and recovery phases. This study aimed to comprehensively profile key biopsychosocial changes occurring pre/post competition in a group of British natural bodybuilders. We measured: resting metabolic rate (RMR), body composition (via BIA, ultrasound and sum of 7 skinfolds), lipid profiles, fasted glucose, blood pressure, power output (Wingate), grip strength, and carried out psychometric questionnaires (EAT-26, STAI, BUT) over an eight-month period. Mean weight loss from 5 months (5M) to pre-contest (PRE) was $8.6 \pm 4.1 \mathrm{~kg}$, followed by an increase of $7.9 \pm 1.6 \mathrm{~kg}$ two months post contest (POST). The mean RMR of the cohort was stable from $1899 \pm 630 \mathrm{kcal}$ (5M) to $1897 \pm 598 \mathrm{kcal}$ (PRE), before decreasing to $1797 \pm 242 \mathrm{kcal}$ (POST). Mean peak power declined from $770 \pm 113.0 \mathrm{w}(5 \mathrm{M})$, to $700.0 \pm 134.4 \mathrm{w}$ (PRE), before increasing to $816.0 \pm 116.2 w$ (POST). Competition dieting had no effect on EAT-26 or BUT scores, suggesting no body dysmorphic or eating disorder concerns, although trait anxiety was elevated in three out of the four participants throughout the observation. Many of the biopsychosocial changes that occur to bodybuilders as they prepare for competition return to baseline measurements 2 months post competition.
\end{abstract}

Key words: physique, body-dysmorphia, drug-free, reverse-diet, bigorexia.

\section{Introduction}

Competitive bodybuilders are judged on muscularity and proportionality. Training and nutrition fall into phases: off-season, competition preparation, peak week and recovery (Chappell and Simper, 2018; Helms et al., 2015; Spendlove et al., 2015). In the off-season bodybuilders gain weight, to add muscle before reducing body fat to increase definition during competition preparation (Chappell and Simper, 2018). Finally, bodybuilders employ peaking strategies immediately prior to competition to 'fine-tune' their appearance (Helms et al., 2014). Contest preparation generally involves 12-32 weeks of energy restriction and increased aerobic activity (Helms et al., 2019; Mäestu et al., 2010). Recently, a 'recovery period' was identified where athletes seek to restore 'normal' hormonal and metabolic function, and resolve any psychological aberrations associated with competition preparation (Mäestu et al., 2010).

Competition preparation's effect on biopsychosocial outcomes is under-researched and longitudinal studies with large cohorts are logistically challenging. Mäestu et al. (2010), however, carried out an 11-week intervention on 14 bodybuilders and Mitchell et al. (2018) recruited 9 bodybuilders for a 20-week case series. Besides these exceptions, most studies are cross sectional and case study designs, sometimes on athletes in categories demanding less muscularity and leanness compared to the bodybuilding, e.g.

\footnotetext{
1 - School of Pharmacy and Life Science, Robert Gordon University, Garthdee Road, Aberdeen, AB10 7AQ, United Kingdom.

2 - Sheffield Hallam University, Department of Food and Nutrition, Pond Street Sheffield S1 1WS, United Kingdom.

3 - Trexler Fitness LLC, Raleigh, NC, United States.

4 - Sports Performance Research Institute New Zealand, Auckland University of Technology, 17 Antares Pl, Rosedale, Auckland 0632, New Zealand.
} 
bikini, figure, or men's physique (Alwan et al., 2019; Trexler et al., 2017). There are, however, numerous single-competitor case studies investigating physiological changes during contest preparation reviewed by Helms et al. (2019) and Alwan et al. (2019). Contest preparation strategies may vary by geographical location and drug testing of the sanctioning federation (Chappell and Simper, 2018); however, we found no case series of natural British bodybuilders. Furthermore, effects of long-term bodybuilding preparation on state and trait anxiety are unknown. A common perception is bodybuilders manipulate their physiques out of a sense of inadequacy known as body dysmorphia or 'bigorexia' (a pathological need to build ever greater muscle mass despite substantial current muscularity); however, its prevalence amongst natural (drug free) bodybuilders is unknown (Devrim et al., 2018). The aim of this investigation was to observe the biopsychosocial changes among a small number of British natural bodybuilders during competition and recovery.

\section{Methods}

\section{Participants}

Four amateur drug-free bodybuilders (3 male), preparing for British Natural Bodybuilding Federation (BNBF) competitions were recruited. The males (P1, P2, and P3) were from the bodybuilding category, and the female participant (P4) was from the figure category. Prior to participating, P2 (44 yrs), and P4 (38 yrs) previously placed in the top 5 at the BNBF finals, P3 (38 yrs) qualified for the finals, and P1 (30 yrs) competed in a regional BNBF qualifier. Participants competed throughout the observation: P1 twice; P3 three times; P4 four times, and P2 five times. All participants were subject to drug testing via urinalysis, and $\mathrm{P} 2$ to $\mathrm{P} 4$ both completed a polygraph prior to the BNBF finals. P1 and P2 managed their own competition preparation, while $\mathrm{P} 3$ and $\mathrm{P} 4$ had coaches.

Participants visited the laboratory every four weeks for eight months, five to six months $(5 \mathrm{M} / 6 \mathrm{M})$ prior to their final competition and 2 months post-competition (POST). The precompetition (PRE) visit was one week before the participant's final competition, with the exception of P3, who visited the lab one week after his final competition. P1 was recruited at the outset of his competition preparation, and P2-P4 six to eight weeks after they started competition preparation. Group mean \pm standard deviation (SD) and individual data are reported. This study was approved by the university ethics committee, in accordance with the Declaration of Helsinki. Participants were informed of all aspects of the study, provided written consent, and gave permission to publish their data after reviewing the final document.

Indirect calorimetry, prediction equations, heart rate and blood pressure

The resting metabolic rate (RMR) was measured per established protocols (Compher et al., 2011). Participants were measured following a $12 \mathrm{~h}$ fast and were asked to refrain from caffeine, physical activity and alcohol for $24 \mathrm{~h}$ preceding visits. Participants rested supine for 20 mins, followed by 10 mins wearing a facemask for RMR measurements (AS Instruments, Oxford, United Kingdom). The RMR was captured by breath-bybreath oxygen $\left(\mathrm{VO}_{2}\right)$ and carbon dioxide $\left(\mathrm{VCO}_{2}\right)$ consumption via Douglas bags and an electronic gas analyzer (GIR250, MTL, Luton, United Kingdom). Gas calibration was carried out using known concentrations $\left(4 \% \mathrm{CO}_{2} ; 16 \% \mathrm{O}_{2}\right.$ and $100 \%$ $\mathrm{N}_{2}$; Spancan MTL, Luton, United Kingdom). Environmental conditions during testing were: humidity $27.9 \pm 7.2 \%$; temperature $22.1 \pm 2.6^{\circ} \mathrm{C}$. The Schofield (Schofield, 1985) and the Cunningham equation (Cunningham, 1980) for estimating the BMR were compared to the measured RMR. Blood pressure and the heart rate were measured using a digital blood pressure monitor (UA-787 Plus, A \& D Instruments Ltd, Oxfordshire, UK) in triplicate with participants supine.

Anthropometry and Body Composition

Participants were asked to consume $500 \mathrm{ml}$ of water the morning of the trial to standardize hydration. Body height was measured using a stadiometer (Holtain, Crymych, United Kingdom) and weight, FM, FFM, and total body water (TBW) were measured via a Tanita multifrequency BIA analyzer (InBody 720, Biospace, Urbandale, Iowa, USA). The BMI and fat free mass index (FFMI) were calculated as $\mathrm{kg} / \mathrm{m}^{2}$ and FFM $\mathrm{kg} / \mathrm{m}^{2}$, respectively. Sum of 7 -site skinfold assessment using amplitude-mode ultrasound (Bodymetrix, Professional Ultrasound, Cartwright fitness, Chester, UK) was used to calculate BF\% 
(Jackson and Pollock, 1978). Ultrasound testing was performed as described by Muller et al. (2016), and was interpreted using Bodymetrix ultrasound analysis software (IntelaMetrix, Brentwood, California, United States).

Blood and saliva measurements

Capillary blood samples were obtained via a single-use lancet (Accu-chek Softclix Pro, Roche Diagnostics Ltd, West Sussex, UK) and microsafe collection tubes (Inverness Medical, Cheshire, UK). Test strips and a reflectance photometer (Reflotron Plus, Inverness Medical) for total cholesterol (TC), whole blood triglycerides (TAG), creatine kinase (CK) and high-density lipoproteins (HDL) were used. The low-density lipoprotein (LDL) fraction was estimated by subtracting the sum of total TAG and HDL divided by 2.19, from TC. Fasting blood glucose (FBG) was measured via whole blood samples added to a heparin and saline solution before analysis by a Biosen C-line monitor (EKF diagnostics, Ebendorfer, Germany). Salivary testosterone and CRP were measured with enzyme immunoassay kits (Salimetrics, Carlsbad, California, USA), prior to analysis on a plate reader at $450 \mathrm{~nm}$ (Synergy HT, BioTek Instruments, Winoski, Vermont, USA), and Gen5 microplate reader software (BioTek Instruments, Winoski, Vermont, USA).

\section{Anaerobic power and hand grip strength}

Anaerobic power was measured via the $20 \mathrm{~s}$ Wingate test as described by Attia et al. (2014) using a Velotron cycle (Racer-Mate, Seattle, WA). Peak, mean, and body mass-scaled power were obtained from Velotron software (RaceMate One V.4.1.0.6). Hand grip strength (HGS) was measured via a hand grip dynamometer (Takei, Nigata City, Nigata Pref, Japan).

Dietary intake and energy availability

The first author (AC; a registered nutritionist) performed $24 \mathrm{~h}$ dietary recalls. Participants documented weights and portions of food and beverages consumed in the previous 24 hours. Participants consumed the same foods across the week and specified where high and low energy days occurred. Data were analyzed using dietary analysis software (Nutritics Research Edition v5.092, Dublin, Ireland). Energy and protein from dietary supplements were calculated per manufacturers' websites. Energy availability (EA) was calculated as total energy intake, divided by FFM, minus an estimate of energy expenditure based on a daily resistance training session between $60-90 \mathrm{~min}$, and cardiovascular exercise between 20-45 min.

Psychological Assessment Questionnaires

The Eating Attitude 26-item questionnaire (EAT-26) (Garner and Garfinkel, 1979) is divided into three subscales: dieting behavior, Bulimia Nervosa and food preoccupation. EAT-26 captures attitudes in the previous 6 months; scores over 20 are regarded as a cause for concern, requiring further assessment. Body dysmorphic disorder (BDD) symptoms were assessed via the Body Uneasiness Test (BUT) (Cuzzolaro et al., 2006) which is a 34-item questionnaire with five subscales assessing weight phobia, body image concern, avoidance, compulsive self-monitoring and depersonalization. BUT scores over 2.0 (global severity index [GSI]) were set as the cutoff for BDD symptoms requiring further assessment. Finally, the State-Trait Anxiety Inventory for Adults (STAI) was used to assess state and trait anxiety. Trait anxiety refers to a relatively stable personality factor indicating anxiety susceptibility, such as perceptions of a stressful situation as dangerous or threatening, while state anxiety refers to transient feelings of stress in a situation (Spielberger and Gorsuch, 1983). The STAI contains two 20-item sets of multiple-choice questions. Scores above 48 and 41 for state and trait anxiety, respectively, were considered 'elevated' (Spielberger and Gorsuch, 1983). All tests were conducted prior to indirect calorimetry.

Design and Procedures and Statistical analysis

This study follows a case-series design which carried out observations for offseason, precompetition and recovery phases of the athletes involved. Descriptive statistics were used to track any alterations to outcome measures between the three phases, these were recorded and analyzed in Microsoft Excel.

\section{Results}

Participants' characteristics and indirect calorimetry Body height of P1 to P4 were 1.76, 1.86, 1.64 , and $1.64 \mathrm{~m}$, respectively. Individual BW, LBM, FM and sum of skinfolds are provided in Table 1 for selected time points and as supplementary Figures (S1). There was a trend from $5 \mathrm{M}$ to PRE of indices decreasing before 
increasing POST. Mean body mass loss $5 \mathrm{M}$ to PRE: $9.0 \pm 3.3 \mathrm{~kg}(10.6 \pm 3.9 \%)$, of which $1.8 \pm 3.0$ $\mathrm{kg}(20.9 \pm 4.4 \%)$ was FFM and $6.8 \pm 1.1 \mathrm{~kg}(79.1 \pm$ $4.5 \%$ FM. Mean body mass regain PRE to POST was $7.5 \pm 1.9 \mathrm{~kg}$, with $1.6 \pm 1.4 \mathrm{~kg}(13.3 \pm 3.3 \%)$ from FFM and $6.9 \pm 2.0 \mathrm{~kg}(87.3 \pm 3.3 \%) \mathrm{FM}$. Except for P1, body mass POST, reflected 5M weight. Mean skinfolds decreased $19.5 \pm 13.5 \mathrm{~mm}$ $5 \mathrm{M}$ to PRE, before increasing PRE to POST by 24.3 $\pm 9.3 \mathrm{~mm}$.

The RMR, TBW, BMI, FFMI, blood pressure, heart rate, blood glucose, blood lipids, CRP, CK and testosterone are presented in Table $1 \mathrm{a} \& \mathrm{~b}$ and as supplementary Figures (S2) for selected time points. There was a trend for the RMR to decrease from $5 \mathrm{M}$ to PRE, before increasing POST. Prediction equations overestimated energy requirements by as much as $911.2 \mathrm{kcal}$ (Schofield) and $727.0 \mathrm{kcal}$ (Cunningham), and under-estimated by as much as $-688.4 \mathrm{kcal}$ (Schofield) and $-617.8 \mathrm{kcal}$ (Cunningham). Mean estimates of energy requirements were $267 \pm 655.0 \mathrm{kcal}$ per day for the Schofield equations and $159.4 \pm 569.6 \mathrm{kcal}$ per day for the Cunningham equations. Waist and hip circumferences and WHR decreased from $5 \mathrm{M}$ to PRE-in all competitors, before increasing POST. The mean waist circumference was: $5 \mathrm{M} 79.4 \pm 7.7$ $\mathrm{cm}$, PRE $72.5 \pm 3.9 \mathrm{~cm}$, POST $79.3 \pm 3.5 \mathrm{~cm}$; hip circumference $5 \mathrm{M}, 101 \pm 4.3 \mathrm{~cm}$, PRE $90.9 \pm 2.8 \mathrm{~cm}$, POST $96.9 \pm 7.9 \mathrm{~cm}$; WHR 5M $0.80 \pm 0.06$, PRE 0.80 \pm 0.4 , POST $0.82 \pm 0.04$.

Dietary intake, exercise regimes, and energy availability

Table 2 displays nutrient intake and a sample participant's diet is provided as a supplement (S3). Foods most commonly consumed were: oats, rice, white and sweet potatoes, poultry, spinach and broccoli. Periodised nutrition was practiced by $\mathrm{P} 3$ and $\mathrm{P} 4$ (where high and low energy was consumed per the training schedule). Daily dietary intake was consistent without "treat" or "cheat meals" with quantities changing to reflect reduced energy intake. Energy declined in competitors' diets 5M to PRE, before increasing PRE to POST (Table $2 a \& b)$, EA followed the same pattern. Participants consumed 1-7 dietary supplements. Whey protein was most frequently used. Maltodextrin, branch chain amino acids, creatine monohydrate, leucine, glutamine, and protein bars were also consumed.
Resistance training was consistent throughout the observation and organized by muscle group e.g. "back" or "leg" day. Participants trained four (P2), five (P1, P4), or six (P3) days per week. Muscle group training frequency reflected relative strengths and weaknesses, participants performed 2 to 5 exercises per muscle group, for 2 to 5 sets. Only P2 reduced training frequency POST, from four to three days per week. P2 and P4 performed fasted aerobics and P1, P2 and P4 performed high intensity interval training (HIIT) 1-3 times per week, for 10-30 min. All participants performed 20-45 min of low intensity steady state (LISS) aerobics 2-13 times per week. The amount of aerobic training increased as participants approached competition before declining in the POST phase.

Performance metrics of bodybuilding competitors

Wingate and HGS results are reported in Table 3a\&b and as a supplement (S4). There was a slight increase in HGS in $\mathrm{P} 3$ and $\mathrm{P} 4$ from $5 \mathrm{M}$ to POST, and a slight decrease in HGS 5M to PRE in P1 and P2. Peak, mean and minimum power declined from $5 \mathrm{M}$ to PRE, before increasing POST, with the exception of P2 who increased mean and minimum power throughout the study.

Eating Attitudes, Body Uneasiness and State Trait Anxiety Testing

Results of EAT-26, STAI and the BUT are presented in Figure 1A-D. EAT-26 scores were between 1-15 (Figure 1A). The dieting subscale contributed most to the overall EAT-26 score (1-9) with item 6: 'I am aware of the calorie content of foods I eat', 19: 'I display self-control around food' and 23: 'I engage in dieting behavior' the highest scoring. Bulimia and oral control scales scored between 06. Participants consistently reported exercising more than $60 \mathrm{~min}$ a day to lose weight, and having lost $9 \mathrm{~kg}$ or more in the past 6 months. In the POST phase, P2 and P4 reported binge-eating at least once monthly.

BUT scores were between 0.2-1.0 (below the 2.0 cut off associated with BDD, see Figure 1B). Weight phobia (0.1-1.2), compulsive selfmonitoring (0.2 to 1.8$)$ and body image concern (0.0-1.2) subscales scored the highest. Avoidance and depersonalization subscales were between 0.0-0.7. State anxiety scored between 20-52, with three occasions where participants scored higher than the 48 cut off (P3: 6M 52; P2: 1M 51, POST 49) 
for elevated state anxiety (Figure 1C). Trait

6M 50) (Figure 1D).

anxiety was between 20-50, only P1 did not exceed the 41-score cut off for elevated anxiety (P2: 4M 50; P3: 5M 47, 4M 46, 1M 42, POST 44: P4

Table 1a

Estimated Energy Requirements and Selected Health Indices of British Natural Bodybuilders

5 Month PRE Competition

\begin{tabular}{|c|c|c|c|c|}
\hline Participant no. & $10^{n}$ & $20^{\pi}$ & $30^{\prime}$ & 49 \\
\hline RMR (kJ) & 5414 & 11652 & 7435 & 7431 \\
\hline RMR (kcal) & 1294 & 2785 & 1777 & 1776 \\
\hline RMR per kg LBM (kcal) & 18.1 & 35.6 & 29.7 & 35.3 \\
\hline Bodyweight (kg) & 85.7 & 87.2 & 72.5 & 63.5 \\
\hline Body Mass Index $\left(\mathrm{kg} / \mathrm{m}^{2}\right)$ & 27.7 & 25.2 & 27.0 & 23.6 \\
\hline Lean Body Mass (kg) & 71.4 & 78.1 & 59.9 & 50.9 \\
\hline Fat Mass (kg) & 14.3 & 9.1 & 12.6 & 12.6 \\
\hline Total Body Water (\%) & 60.9 & 64.7 & 60.7 & 60.0 \\
\hline$\Sigma$ Skin Fold Thickness (mm) & 50 & 29 & 30 & 59 \\
\hline Bodyfat \% (BIA) & 16.7 & 10.4 & 17.4 & 19.8 \\
\hline SYS Blood Pressure (mmHg) & 122 & 128 & 99 & 115 \\
\hline DIA Blood Pressure (mmHg) & 74 & 80 & 69 & 66 \\
\hline Resting Heart Rate (bpm) & 56 & 53 & 63 & 58 \\
\hline Fasting Blood Glucose (mM) & 4.8 & 5.1 & 3.4 & 3.0 \\
\hline Total Cholesterol (mM) & 3.9 & 4.1 & 2.9 & 3.9 \\
\hline HDL (mM) & 0.9 & 1.1 & - & 1.1 \\
\hline LDL (mM) & 0.9 & 1.0 & - & 0.7 \\
\hline Triglycerides (mM) & 1.0 & 0.9 & 0.8 & 0.8 \\
\hline Testosterone (pg/ml) & 114.6 & 208.3 & 107.6 & 38.2 \\
\hline C-Reactive Protein (ng/ml) & 896.9 & 1298.4 & 17.7 & 17.1 \\
\hline Creatine Kinase (I/U) & 103.0 & 196.0 & 44.7 & 90.8 \\
\hline
\end{tabular}

$\$$ one week pre shows carb depleting, first bodybuilding shows. *one day pre shows carb loading, $¥$ Lean body mass based on BIA, SYS systolic, DIA diastolic, BPM beats per minute, LDL Low density lipoprotein, HDL high density lipoprotein, - measurement not recorded. o' male, I female, LBM lean body mass, BMI body mass index, FFMI fat free mass index 
Table 1b

Estimated Energy Requirements and Selected Health Indices of British Natural Bodybuilders

\begin{tabular}{|c|c|c|c|c|c|c|c|c|}
\hline \multirow[b]{2}{*}{ Participant no. } & \multicolumn{4}{|c|}{ PRE Competition } & \multicolumn{4}{|c|}{ POST Competition } \\
\hline & $10^{\pi}$ & $20^{\pi}$ & $30^{\pi}$ & $4 \% *$ & $10^{x}$ & $20^{7}$ & $30^{x}$ & 4 우 \\
\hline RMR (kJ) & 6402 & 10824 & 5368 & 9150 & 7556 & 8916 & 7008 & 6586 \\
\hline RMR (kcal) & 1530 & 2587 & 1283 & 2187 & 1806 & 2131 & 1675 & 1574 \\
\hline RMR per kg LBM (kcal) & 23.4 & 33.0 & 21.2 & 44.8 & 26.3 & 27.6 & 27.9 & 30.9 \\
\hline Bodyweight (kg) & 71.9 & 80.8 & 66.3 & 57.0 & 79.1 & 87.4 & 72.2 & 67.2 \\
\hline Body Mass Index $\left(\mathrm{kg} / \mathrm{m}^{2}\right)$ & 23.2 & 23.1 & 24.4 & 21.2 & 25.5 & 25.0 & 26.5 & 25.0 \\
\hline Lean Body Mass (kg) & 65.4 & 78.4 & 60.6 & 48.8 & 68.7 & 77.1 & 60.0 & 50.9 \\
\hline Fat Mass (kg) & 6.5 & 2.4 & 5.7 & 8.2 & 22.9 & 22.3 & 23.0 & 18.9 \\
\hline Total Body Water (\%) & 65.2 & 72.4 & 67.0 & 63.0 & 62.8 & 62.9 & 60.9 & 57.3 \\
\hline$\Sigma$ Skin Fold Thickness (mm) & 24 & 15 & 27 & 37 & 35 & 40 & 49 & 63 \\
\hline Bodyfat \% (BIA) & 9.1 & 3.0 & 8.6 & 14.3 & 13.2 & 11.8 & 16.9 & 24.2 \\
\hline SYS Blood Pressure (mmHg) & 109 & 117 & 97 & 113 & 110 & 126 & 99 & 120 \\
\hline DIA Blood Pressure (mmHg) & 68 & 72 & 65 & 70 & 68 & 83 & 69 & 67 \\
\hline Resting Heart Rate (bpm) & 54 & 52 & 55 & 41 & 55 & 60 & 69 & 56 \\
\hline Fasting Blood Glucose (mM) & 4.8 & 4.6 & 3.7 & 4.0 & 4.9 & 5.0 & 4.1 & 4.4 \\
\hline Total Cholesterol (mM) & 4.8 & 4.6 & 3.7 & 4.0 & 4.8 & 5.8 & 4.5 & 3.9 \\
\hline HDL (mM) & 1.5 & 0.9 & 0.8 & 1.4 & 1.0 & 1.0 & 0.9 & 1.5 \\
\hline LDL (mM) & 1.2 & 1.1 & 1.2 & 0.6 & 1.4 & 1.8 & 1.1 & 0.7 \\
\hline Triglycerides (mM) & 1.3 & 0.8 & 0.8 & 0.8 & 0.8 & 0.8 & 0.12 & 0.8 \\
\hline Testosterone (pg/ml) & 68.9 & 50.2 & 87.9 & 66.1 & 159.7 & 283.7 & 126.9 & - \\
\hline C-Reactive Protein (ng/ml) & 26.0 & 1292.7 & 38.3 & 163.1 & 37.6 & 900.5 & 65.9 & - \\
\hline Creatine Kinase (I/U) & 200.0 & 720.0 & 227.0 & 91.6 & 346.0 & 339.0 & 130.0 & 121.0 \\
\hline
\end{tabular}

\$ one week pre shows carb depleting, first bodybuilding shows. ${ }^{*}$ one day pre shows carb loading, ¥ Lean body mass based on BIA, SYS systolic, DIA diastolic, BPM beats per minute, LDL Low density lipoprotein, HDL high density lipoprotein, - measurement not recorded. ơ male, o female, LBM lean body mass, BMI body mass index, FFMI fat free mass index 
Table 2a

$24 \mathrm{~h}$ dietary intake of Bodybuilders Preparing for Competition

\begin{tabular}{|c|c|c|c|c|c|c|c|c|}
\hline \multirow[b]{2}{*}{ Participant no. } & \multicolumn{4}{|c|}{5 month PRE Competition } & \multicolumn{4}{|c|}{1 month PRE Competition } \\
\hline & $10^{7}$ & $2 \sigma^{7}$ & $3 \sigma^{7}$ & 49 & $10^{n}$ & $2 \sigma^{7}$ & $30^{n}$ & $4 ㅇ$ \\
\hline \multicolumn{9}{|c|}{ Total Macronutrient Intake } \\
\hline $\mathrm{CHO}(\mathrm{g})$ & 320 & 422 & 358 & 249 & 196 & 380 & 236 & 187 \\
\hline Fibre (g) & 31 & 46 & 35 & 27 & 15.7 & 26.4 & 41 & 34.2 \\
\hline PRO (g) & 212 & 224 & 182 & 147 & 186 & 192 & 207 & 172 \\
\hline Fat (g) & 44 & 73 & 63 & 37 & 53 & 26.5 & 49 & 35.7 \\
\hline Energy (kJ) & 10540 & 13569 & 11447 & 8054 & 8372 & 10577 & 9238 & 7372 \\
\hline Energy (kcal) & 2519 & 3243 & 2736 & 1925 & 2001 & 2528 & 2208 & 1762 \\
\hline
\end{tabular}

Intake Scaled for BW

\begin{tabular}{|c|c|c|c|c|c|c|c|c|}
\hline CHO (g/kg BW) & 3.7 & 4.9 & 4.9 & 3.9 & 2.6 & 4.8 & 3.7 & 3.2 \\
\hline PRO (g/kg BW) & 2.5 & 2.6 & 2.5 & 2.3 & 2.5 & 2.4 & 3.2 & 2.9 \\
\hline Fat (g/kg BW) & 0.5 & 0.9 & 0.9 & 0.6 & 0.7 & 0.3 & 0.8 & 0.6 \\
\hline Energy (kcal/kg BW) & 29.4 & 38.2 & 37.7 & 30.3 & 26.7 & 31.8 & 34.2 & 30.0 \\
\hline
\end{tabular}

Intake scaled for LBM

$\begin{array}{lllllllll}\text { Protein (g/kg LBM) } & 3.0 & 2.8 & 3.0 & 2.9 & 2.8 & 2.5 & 3.4 & 3.5 \\ \text { Energy Availability (kcal/kg LBM)* } & 26.9 & 32.8 & 35.7 & 26.0 & 20.8 & 25.0 & 26.7 & 23.7\end{array}$

\section{Percentage of Energy}

$\begin{array}{lcccccccc}\text { CHO (\%) } & 50.8 & 54.6 & 52.7 & 52.1 & 39.1 & 60.2 & 41.6 & 44.3 \\ \text { PRO (\%) } & 33.6 & 26.7 & 26.6 & 30.8 & 37.2 & 30.4 & 38.5 & 37.6 \\ \text { Fat (\%) } & 15.7 & 18.7 & 20.7 & 17.1 & 23.7 & 9.4 & 19.9 & 18.1\end{array}$

Intake from Supplements

$\begin{array}{lllllllll}\text { PRO (\%) } & 21.4 & 36.4 & 36.4 & 33.1 & 37.9 & 36.7 & 21.0 & 35.1 \\ \text { Energy (\%) } & 8.9 & 10.9 & 19.3 & 12.9 & 18.0 & 23.2 & 9.6 & 17.5\end{array}$

*Energy availability based on a hypothetical energy expenditure of $600 \mathrm{kcal}$ per day. Abbreviations, $\mathrm{E}$ male, \& female, CHO Carbohydrate, PRO Protein, BW body weight, LBM lean body mass calculated by bioelectrical impedance - measurement not recorded 
Table 2b

$24 \mathrm{~h}$ dietary intake of Bodybuilders Preparing for Competition

\begin{tabular}{|c|c|c|c|c|c|c|c|c|}
\hline \multirow[b]{2}{*}{ Participant no. } & \multicolumn{4}{|c|}{ PRE Competition } & \multicolumn{4}{|c|}{ POST Competition } \\
\hline & $10^{x}$ & $20^{\pi}$ & $30^{x}$ & 49 & $10^{x}$ & $20^{x}$ & $30^{x}$ & 49 \\
\hline \multicolumn{9}{|c|}{ Total Macronutrient Intake } \\
\hline $\mathrm{CHO}(\mathrm{g})$ & 228 & 426 & 222 & 182 & 346 & 333 & 369 & 305 \\
\hline Fibre (g) & 25 & 28 & 40 & 27 & 33 & 39 & 38 & 35 \\
\hline PRO (g) & 189 & 171 & 166 & 145 & 193 & 187 & 222 & 188 \\
\hline Fat (g) & 51 & 30 & 38 & 27 & 67 & 80 & 45 & 44 \\
\hline Energy (kJ) & 8933 & 11134 & 7908 & 6498 & 11573 & 11698 & 11590 & 9874 \\
\hline Energy (kcal) & 2135 & 2661 & 1890 & 1553 & 2766 & 2796 & 2770 & 2360 \\
\hline
\end{tabular}

Intake Scaled for BW

$\begin{array}{lcccccccc}\text { CHO (g/kg BW) } & 3.2 & 5.3 & 3.3 & 3.2 & 4.4 & 3.8 & 5.1 & 4.5 \\ \text { PRO (g/kg BW) } & 2.6 & 2.1 & 2.5 & 2.5 & 2.4 & 2.1 & 3.1 & 2.8 \\ \text { Fat }(\mathrm{g} / \mathrm{kg} \mathrm{BW}) & 0.7 & 0.4 & 0.6 & 0.5 & 0.9 & 0.9 & 0.6 & 0.6 \\ \text { Energy (kcal/kg BW) } & 29.7 & 32.9 & 28.5 & 27.2 & 35.0 & 32.0 & 38.4 & 35.1\end{array}$

Intake scaled for LBM

$\begin{array}{lcccccccc}\text { Protein (g/kg LBM) } & 2.9 & 2.2 & 2.7 & 3.0 & 2.8 & 2.4 & 3.7 & 3.7 \\ \text { Energy Availability (kcal/kg LBM)* } & 23.5 & 26.3 & 21.3 & 19.5 & 31.5 & 28.5 & 36.2 & 34.5\end{array}$

\section{Percentage of Energy}

$\begin{array}{lcccccccc}\text { CHO (\%) } & 42.2 & 64 & 46.9 & 42.2 & 49.9 & 42.2 & 42.2 & 42.2 \\ \text { PRO (\%) } & 36.8 & 25.7 & 35.1 & 36.8 & 28.1 & 36.8 & 36.8 & 36.8 \\ \text { Fat (\%) } & 21.1 & 10.3 & 18 & 21.1 & 22.1 & 21.1 & 21.1 & 21.1\end{array}$

Intake from Supplements

\begin{tabular}{lllllllll} 
PRO (\%) & 24.9 & 27.5 & 14.2 & 28.8 & 12.2 & 22.4 & 37.9 & 14.4 \\
Energy (\%) & 11.2 & 13.3 & 6.3 & 14.0 & 4.3 & 7.9 & 18.0 & 11.6 \\
\hline
\end{tabular}

*Energy availability based on a hypothetical energy expenditure of $600 \mathrm{kcal}$ per day. Abbreviations, E male, o female, CHO Carbohydrate, PRO Protein, BW body weight,

LBM lean body mass calculated by bioelectrical impedance - measurement not recorded 
Table 3a

Performance metrics for bodybuilding

\begin{tabular}{|c|c|c|c|c|c|c|c|c|}
\hline \multirow[b]{2}{*}{ Participant no. } & \multicolumn{4}{|c|}{5 month PRE Competition } & \multicolumn{4}{|c|}{1 month PRE Competition } \\
\hline & $10^{\pi}$ & $20^{\pi}$ & $30^{\prime \prime}$ & 4 우 & $10^{\pi}$ & $20^{\pi}$ & $30^{\pi}$ & 49 \\
\hline \multicolumn{9}{|l|}{ Hand Grip Strength } \\
\hline Left (kgf) & 59.4 & 56.7 & 33.3 & 20.7 & 58.2 & 56.8 & 32.9 & 25.1 \\
\hline Right (kgf) & 67.5 & 68.5 & 33.0 & 19.1 & 59.0 & 59.2 & 40.1 & 22.7 \\
\hline \multicolumn{9}{|l|}{ Wingate } \\
\hline Peak Watts (W) & 841 & 863. & 760 & 614 & 742 & 831 & 650 & 533 \\
\hline An. Power (W/kg BW) & 9.8 & 9.9 & 10.5 & 9.7 & 9.9 & 10.5 & 10.1 & 9.1 \\
\hline Mean Watts (W) & 772 & 729 & 633 & 507 & 674 & 746 & 544 & 461 \\
\hline An. Capacity (W/kg BW) & 9.0 & 8.4 & 8.7 & 8.0 & 9.0 & 9.4 & 8.4 & 7.8 \\
\hline Min Watts (W) & 675 & 497 & 540 & 394 & 574 & 630 & 415 & 376 \\
\hline Fatigue Index (\%) & 13.3 & 33.3 & 11.6 & 11.4 & 11.3 & 15.0 & 13.4 & 8.1 \\
\hline Total Work (J) & 15449 & 14580 & 12668 & 10137 & 13481 & 14926 & 10881 & 9216 \\
\hline
\end{tabular}

Table 3b

Performance metrics for bodybuilding

\begin{tabular}{|c|c|c|c|c|c|c|c|c|}
\hline \multirow[b]{2}{*}{ Participant no. } & \multicolumn{4}{|c|}{ PRE Competition } & \multicolumn{4}{|c|}{ POST Competition } \\
\hline & $10^{x}$ & $20^{7}$ & $30^{7}$ & 49 & $10^{x}$ & $20^{x}$ & $30^{x}$ & 49 \\
\hline \multicolumn{9}{|l|}{ Hand Grip Strength } \\
\hline Left (kgf) & 55.2 & 62.3 & 35.9 & 26.0 & 57.6 & 59.1 & 35.8 & 21.9 \\
\hline Right (kgf) & 65.1 & 59.3 & 41.8 & 28.4 & 63.0 & 66.9 & 47.6 & 22.4 \\
\hline \multicolumn{9}{|l|}{ Wingate } \\
\hline Peak Watts (W) & 721 & 852 & 700 & 525 & 858 & 937 & 808 & 661 \\
\hline An. Power (W/kg BW) & 10.0 & 10.5 & 10.6 & 9.2 & 10.6 & 10.7 & 11.2 & 9.8 \\
\hline Mean Watts (W) & 659 & 765 & 572 & 426 & 758 & 840 & 687 & 530 \\
\hline An. Capacity (W/kg BW) & 9.2 & 9.5 & 8.6 & 7.8 & 9.6 & 9.6 & 9.5 & 7.9 \\
\hline Min Watts (W) & 557 & 664 & 441 & 350 & 613 & 706 & 539 & 391 \\
\hline Fatigue Index (\%) & 11.1 & 14.9 & 14.8 & - & 13.7 & 15.8 & 14.8 & 13.6 \\
\hline Total Work (J) & 13179 & 15291 & 10922 & - & 15159 & 16808 & 13741 & 10597 \\
\hline \multicolumn{9}{|c|}{$\begin{array}{c}\text { Abbreviations. Kgf kilograms of force, An. Anaerobic, W watts, J joules, - no data, Min } \\
\text { minimum, no. number, o'Male, of Female }\end{array}$} \\
\hline
\end{tabular}



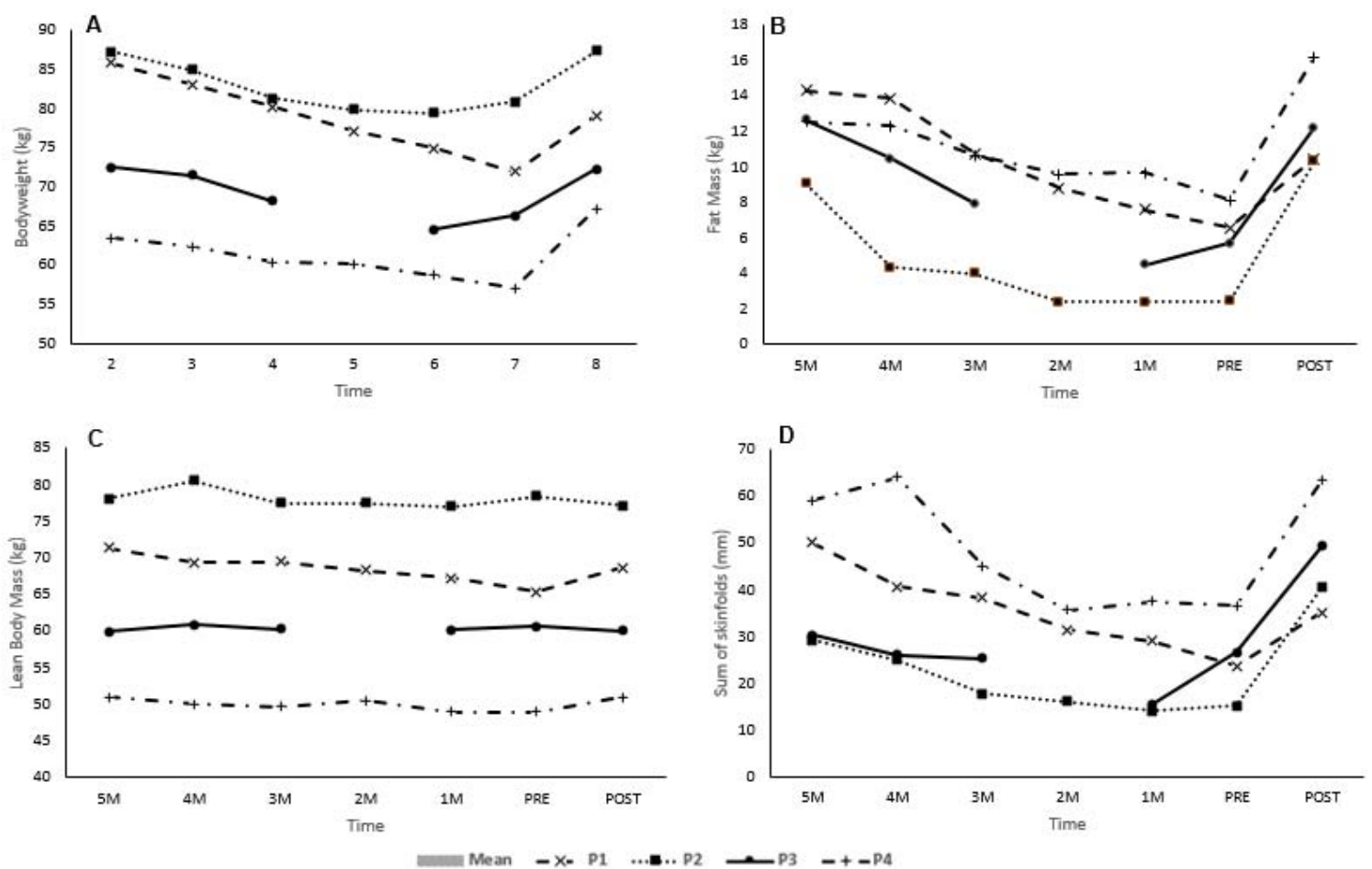

Figure 1A-D.

Psychological Assessment of Competitive Natural Bodybuilders throughout Competition

Preparation. A Eating Attitudes 26 (concern score cut off 20), B Body Uneasiness Test (body dysmorphic disorder concern cut off 2.0), C State Trait Anxiety Index, State Test (elevated state anxiety cut off 48), D State Trait Anxiety Index, Trait Test (elevated trait anxiety cut off 41). Abbreviations. $M$ months from final competition, PRE final visit to the lab prior to the last competition of the season, POST 8 weeks post the PRE laboratory visit.

\section{Discussion}

This study documents biopsychosocial changes of 4 bodybuilders during competition preparation and recovery. As participants lost body mass, metabolic indices of health (FBG, blood lipids, blood pressure) improved, while testosterone and anaerobic performance decreased, and HGS was largely unaffected. This is the first case series performed on a British natural bodybuilding cohort, and the first to report bodybuilders' eating/body image attitudes and anxiety during competition preparation. Overall, there were few effects on eating and body image attitudes, although we observed elevated state and trait anxiety.

All participants reduced their mass during competition preparation. The $10.6 \%$ weight loss is consistent with the 11.1 and $13.2 \%$ weight loss previously reported amongst British natural bodybuilders (Chappell and Simper, 2018). Moreover, $20.9 \%$ of body mass losses from FFM is consistent with the "one-quarter rule" 
documented in a weight loss study (Heymsfield et al., 2014), and the $20.1 \%$ FFM loss observed in a bodybuilding case series observation by Rossow et al. (2013). Interindividual variation in FFM losses is important to note; 43.7 and $32.0 \%$ of all weight lost by $\mathrm{P} 1$ and $\mathrm{P} 4$ was FFM compared to an increase in FFM of 3.8 and $11.5 \%$ in P2 and P3, respectively. Weight loss as high as $43 \%$ from FFM was reported previously by Robinson et al. (2015), while Petrizzo et al. (2017) reported no FFM loss in their natural bodybuilding case study. Thus, some competitors may be less or more likely to lose FFM than others due to individual factors.

Identifying strategies to preserve muscle mass during dieting is important for bodybuilders. Protein intake between 2.3-3.3 g/kg BW of LBM is recommended to maintain muscle during contest preparation (Helms, 2014). Participants 1 and 4 consumed at least $2.3 \mathrm{~g} / \mathrm{kg}$ BW LBM throughout their diets, yet reported the greatest FFM losses. This suggests overall energy intake may play a more important role in preserving FFM. Indeed, Fagerberg (2018) proposed avoiding chronically low energy availability (LEA) with a cut-off of $30 \mathrm{kcal} / \mathrm{kg}$ FFM/day in females and $25 \mathrm{kcal} / \mathrm{kg}$ FFM/day in males, as chronic LEA may result in negative biopsychosocial effects including disproportionate FFM losses (Fagerberg et al., 2018). P1 and P4 had the lowest estimated EA (19.5 to $26.9 \mathrm{kcal} / \mathrm{kg}$ FFM/day), which might explain some of the FFM loses compared to P2 and P3 (estimated EA between 21.3 and $35.7 \mathrm{kcal} / \mathrm{kg}$ FFM/day $5 \mathrm{M}$ to PRE). However, this is speculative given the length of time spent below the Fagerberg thresholds rather than the estimated average energy intake may be important. Moreover, the limitations of dietary assessment and EA estimations should be considered (Shim et al., 2014). These results justify the need for individualized dietary and training strategies to minimize FFM losses.

Bodybuilding case studies previously reported the metabolic rate, thyroid hormone, testosterone, oestrogen, and leptin suppression, and elevated ghrelin during prolonged dieting (Mäestu et al., 2010). We observed that the testosterone level in the male cohort decreased from $5 \mathrm{M}$ to the PRE-levels before returning to $5 \mathrm{M}$ levels POST, coinciding with the increase in FM, energy availability and anaerobic performance. Interestingly, testosterone levels returned to $5 \mathrm{M}$ levels during POST for P1 despite failing to regain all previous weight. Purportedly, low-fat diets negatively affect testosterone and adrenal androgen concentrations compared to isocaloric high-fat diets (Dorgan et al., 1996; Hämäläinen et al., 1984). All participants were low-fat dieters $(0.3$ to $0.8 \mathrm{~g}$ per $\mathrm{kg} \mathrm{BW})$, and increased energy intake POST competition via protein, carbohydrate and fats; thus, direct links between fat intake and restoration of normal hormonal function may be speculative in bodybuilders. Finally, supplement intake was similar to British Professional natural bodybuilders (Chappell and Simper, 2018), with $4-24 \%$ of energy and $12-38 \%$ of protein energy coming from supplements.

The RMR decreased during the dieting phase before increasing POST in 3 of 4 participants relative to $1 \mathrm{M}$. This increase in the PRE-phase may reflect an increase in metabolism during peaking plans the week prior to competition. The reduction in the RMR, however, is consistent with reports in dieting bodybuilders where Robinson et al. (2015) noted an $200 \mathrm{kcal}$ drop in requirements, while the restoration of the RMR might be explained by a return in leptin levels, thyroid function, and body mass reported previously (Alwan et al., 2019; Trexler et al., 2017). The RMR varied significantly compared to prediction equations which may be explained by prior days of exercise and energy intake, highlighting difficulties in capturing information from competitive athletes (Speakman and Sellman, 2003). Participants were normotensive, and blood pressure, and the HR decreased $5 \mathrm{M}$ to PRE mirroring findings from Hulmi et al. (2017). This decrease is probably caused by improved aerobic fitness, although a decrease associated with overtraining was proposed by Rossow et al. (2013). Blood pressure and the HR increased during POST, which is consistent with previous reports. All competitors' FBG, CRP and blood lipids were within reference ranges, while $\mathrm{CK}$ was similar to other resistance training populations and within healthy limits (Baird et al., 2013).

Absolute and relative anaerobic power and capacity increased initially before declining $5 \mathrm{M}$ to PRE, then increasing POST. The initial increase may be a training effect of increased exercise efficiency; however, being extremely lean 
and in an energy deficit likely harmed performance despite improvements in the lean:fat mass ratio. Similar performance trends were observed among natural bodybuilders which resolved post competition (Robinson et al., 2015; Rohrig et al., 2017; Rosso et al., 2013; Trexler et al., 2017; Tinsley et al., 2018). Although, it is worth noting that not all performance variables declined, as HGS was unaffected. Furthermore, Hulmi et al. (2017) noted no decline in explosive and lower body strength during competition preparation.

No participants displayed eating disorder or BDD symptoms according to our questionnaires. EAT-26 scores were similar to a recent cross section of natural bodybuilders (Mitchell et al., 2017a). A meta-analysis, however, reported higher prevalence of eating disorder and BDD symptoms in bodybuilders compared to recreational exercisers (Mitchell et al., 2017b). Authors of a recent review on competitive bodybuilding strategies suggested that personality traits, in particular neuroticism, may be important factors in determining food and body image attitudes (Helms et al., 2019). Similarly to Tinsley et al. (2018), the present participants monitored their physiques and nutrition continuously and practiced restrained eating, without displaying characteristics of emotional eating. It might be more useful for researchers to develop bodybuilding-specific metrics, given the unique dietary perspectives and practices in competitive bodybuilding.

State anxiety was elevated on two occasions in P2 and P4, while their trait anxiety was elevated throughout the observation. Whether elevated anxiety was due to competition dieting, or this is simply a population with high trait anxiety is unknown. Cross sections of competitive bodybuilders have previously reported psychological distress with elevated anxiety, anger and short temper in male and female competitors (Andersen et al., 1985, 1998). Moreover, changes in mood states and psychological distress associated with competition dieting were also reported in case studies of bodybuilders (Robinson et al., 2013; Rohrig et al., 2017). In contrast, Hulmi et al. (2017) noted no change in mood states among 30 physique-sport competitors pre, during and post competition compared to controls.

\section{Strengths and Limitations}

Participants underwent urinalysis and polygraph testing in an effort to ensure natural status. Although we observed a small cohort, this allowed for detailed observations. While measurement timing was standardized relative to competition dates, outcomes were inherently influenced by acute magnitudes of energy deficiency and fatigue, and the body composition measurement techniques have known shortcomings. Throughout contest preparation, specific phases of training are particularly arduous as a result of training volume or energy restriction, which potentially influenced the results. Although we directed participants to attend testing fasted and abstain from exercise, the participants' training schedule meant this was not always precise. Moreover, the psychological questionnaires were validated in nonbodybuilding populations, so their utility in bodybuilders requires further inquiry. Furthermore, additional markers of stress such as the hormone cortisol would have been useful to support the questionnaire data. Finally, performance was tested in a fasted state, which likely impacted our ability to recruit participants.

\section{Conclusions and Reflections}

P2 and P3 placed top 5 at the British finals, while P4 did not. P1 competed in two regional qualifying competitions, but failed to place. In the first author's opinion (an international bodybuilding judge), P2-P4 were competent amateurs who provided insights into how British natural bodybuilders prepared for competition. Regarding best practices, all competitors consumed sufficient protein $(>2.3$ $\mathrm{g} / \mathrm{kg} \mathrm{BW)}$ and dieted over a 6-month period achieving a $0.5-1.0 \%$ bodyweight loss weekly to promote FFM retention as per published guidelines (Helms et al., 2014). As a future strategy to better retain FFM, athletes may benefit from higher energy availability (> $30 \mathrm{kcal} / \mathrm{kg} \mathrm{BW}$ ) by either manipulating their training load or increasing energy intake. Planned diet breaks and refeeds to reduce time spent in a state of LEA should also be considered. Finally, rapid bodyweight and bodyfat regain seen by competitors in this study likely aided prompt recovery of physiological variables.

Researchers should focus on recruiting larger cohorts to determine if higher energy diets 
with sufficient protein are more effective at preserving FFM during calorie restriction. Furthermore, research determining optimal recovery of hormonal and physiological function is needed; specifically, if rapid compared to gradual weight regain is ideal. Finally, further assessment of the psychological effects of competition preparation in larger cohorts is needed to confirm or contradict our findings.

\section{Acknowledgements}

AJC, ETT and ERH earn income as writers and practitioners within the fitness industry. We would like to acknowledge the participants' time and effort in taking part in this study.

\section{Supplementary Information}

\section{Supplement 1}
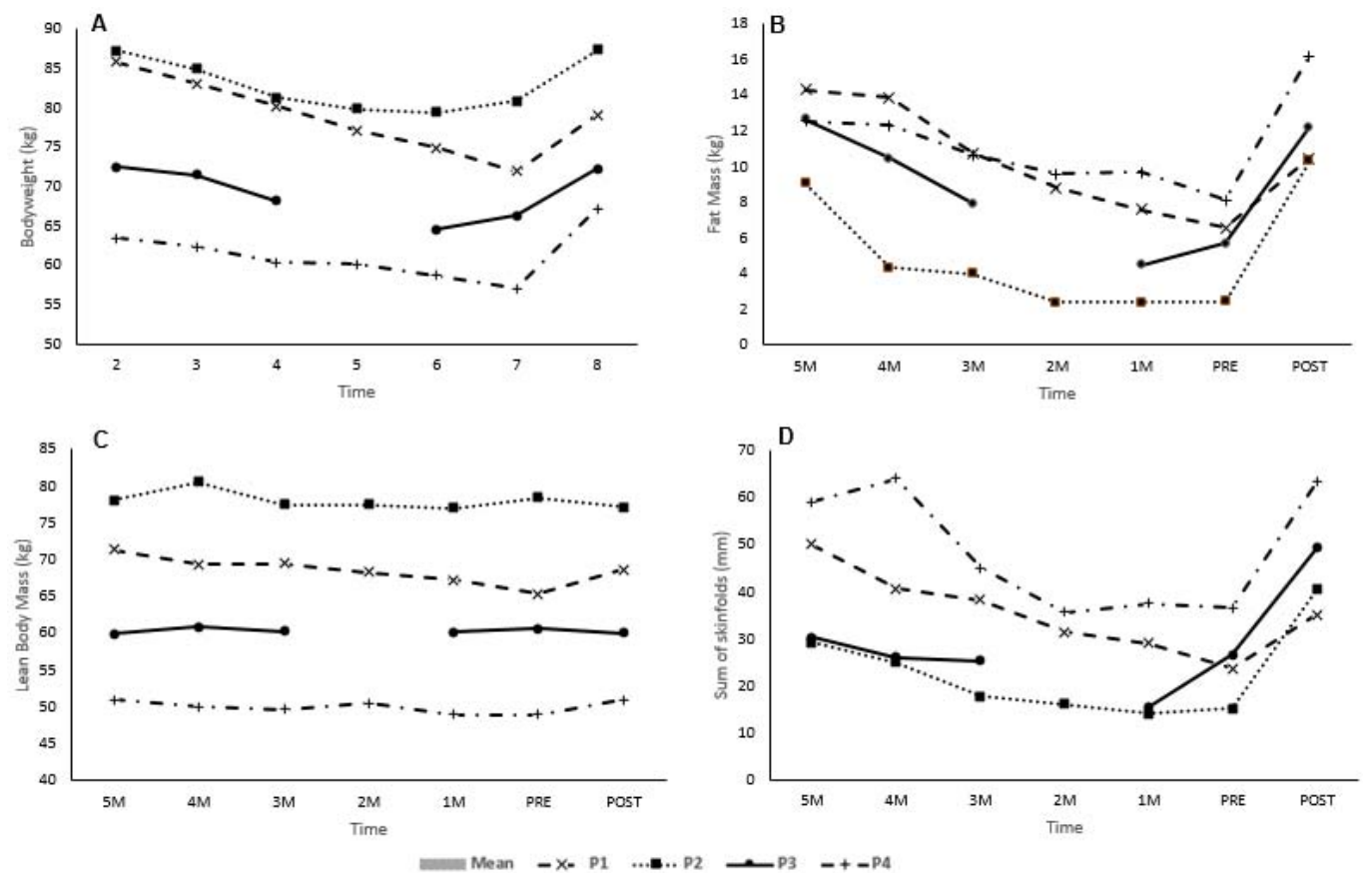

Supplementary Figure (S1) A-D. Changes in Bodybuilders Body Mass during Competition Preparation. A Bodyweight, $\boldsymbol{B}$ Fat mass, $\boldsymbol{C}$ Lean body mass, $\boldsymbol{D}$ Sum of skin folds. Abbreviations. M months from final competition, PRE-final visit to the lab prior to the last competition of the season, POST 8 weeks post the PRE laboratory visit. 


\section{Supplement 2}
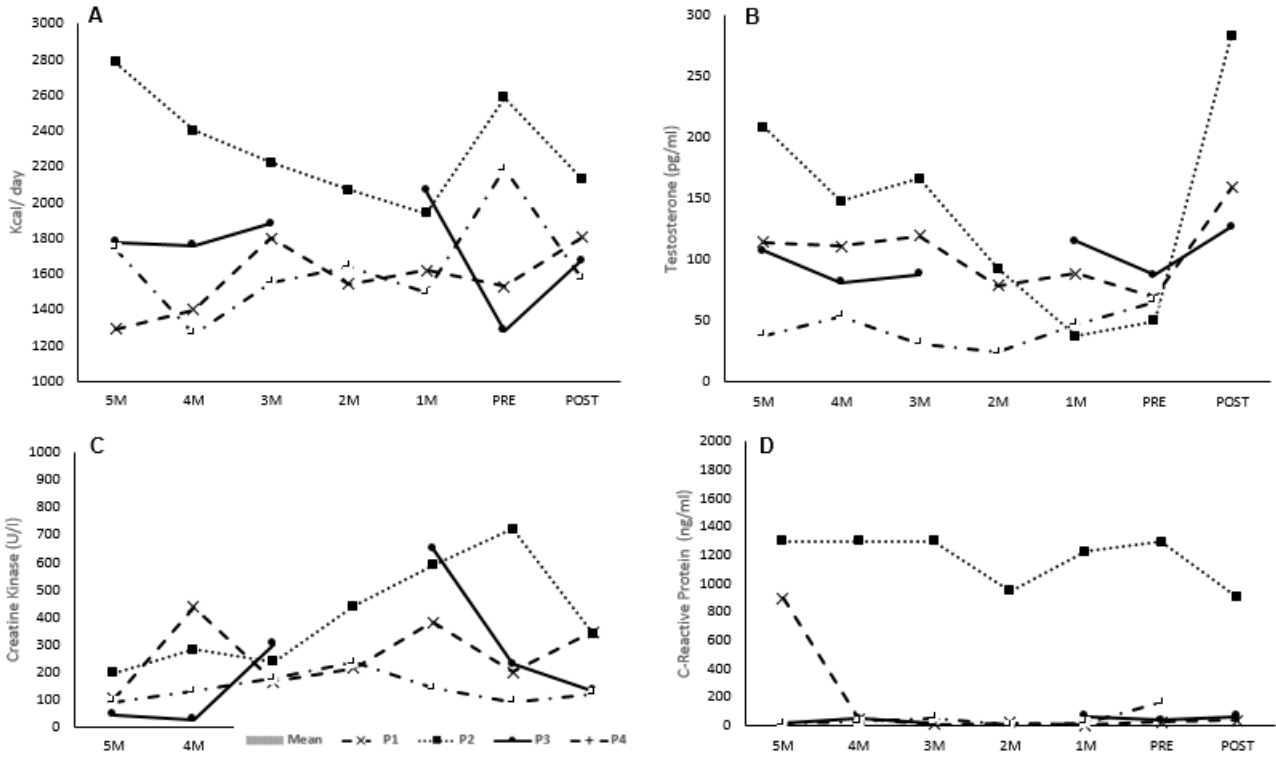

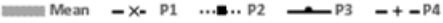

Supplementary Figure (S2) A-D. Change in Bodybuilders RMR and Selected Health Biomarkers during Competition Preparation. A Resting metabolic rate, B Testosterone, C Creatine kinase, D C-Reactive Protein. Abbreviations. M months from final competition, PRE final visit to the lab prior to last competition of the season, POST 8 weeks post the PRE laboratory visit.

\section{Supplement 3}

Supplement Table (S3). Participant 4, Sample Bodybuilding Menu (High Calorie Day) 6 Months from Competition

\begin{tabular}{cc}
\hline & Item/description \\
\hline Meal 1 & \\
& Porridge Oats \\
& Blueberries \\
& Eggs \\
& Spinach \\
& Mushrooms \\
& Onion White \\
& Leucine \\
& Glutamine \\
& \\
Meal 2 & Chicken Breast \\
& Sweet Potato \\
& Spinach
\end{tabular}

Meal 3

Chicken Breast
White Basmati Rice
Almonds

Beverages

Amount $(\mathrm{g})$
$40 \mathrm{~g}(1$ serving, $1 / 2$ cup $)$
$40 \mathrm{~g}(15$ Blueberries $)$
$200 \mathrm{~g}(6,1$ Whole, 5 Whites $)$
$25 \mathrm{~g}$ (tablespoon $)$
$70 \mathrm{~g}(1$ cup $)$
$40 \mathrm{~g}(3$ slices $)$
$4 \mathrm{~g}(1$ teaspoon $)$
$5 \mathrm{~g}(1$ teaspoon $)$

$50 \mathrm{~g}$

$200 \mathrm{~g}\left(1^{1 / 2}\right.$ small $)$ $50 \mathrm{~g}$ (2 tablespoon)

$\begin{array}{rc}\text { Meal 4 } & \text { Item/description } \\ & \text { Chicken Breast } \\ & \text { Sweet Potato } \\ \text { Mixed Frozen Vegetab } & \\ \text { Meal } 5 & \\ & \text { Salmon, Baked } \\ & \text { Sweet Potato } \\ & \text { Spinach } \\ \text { Meal 6 - Post Exercise } \\ \text { Whey Protein } \\ \text { Coco Pops } \\ \text { Leucine }\end{array}$

Meal 7

$$
\begin{gathered}
\text { Porridge Oats } \\
\text { Casein Protein } \\
\text { Almonds }
\end{gathered}
$$

Amount (g)

$75 \mathrm{~g}$

$200 \mathrm{~g}\left(1 \frac{1}{2}\right.$ small $)$

$100 \mathrm{~g}(0.6$ cup $)$

$$
\begin{gathered}
50 \mathrm{~g} \\
200 \mathrm{~g}\left(1 \frac{112 \text { small })}{50 \mathrm{~g}(2 \text { tablespoon })}\right.
\end{gathered}
$$

$50 \mathrm{~g}(1 / 2$ cup $)$
$20 \mathrm{~g}(19$ whole $)$

Black Coffee (4 cups), Pepsi Max (1 can)

\begin{tabular}{ccccc}
\hline \multicolumn{5}{c}{ Total Energy and Macronutrient Intake } \\
\hline Carbohydrates (g) & Fibre (g) & Protein (g) & Fat (g) & Energy (kJ/kcal) \\
295 & 41 & 189 & 52 & 10083,2410 \\
\hline
\end{tabular}




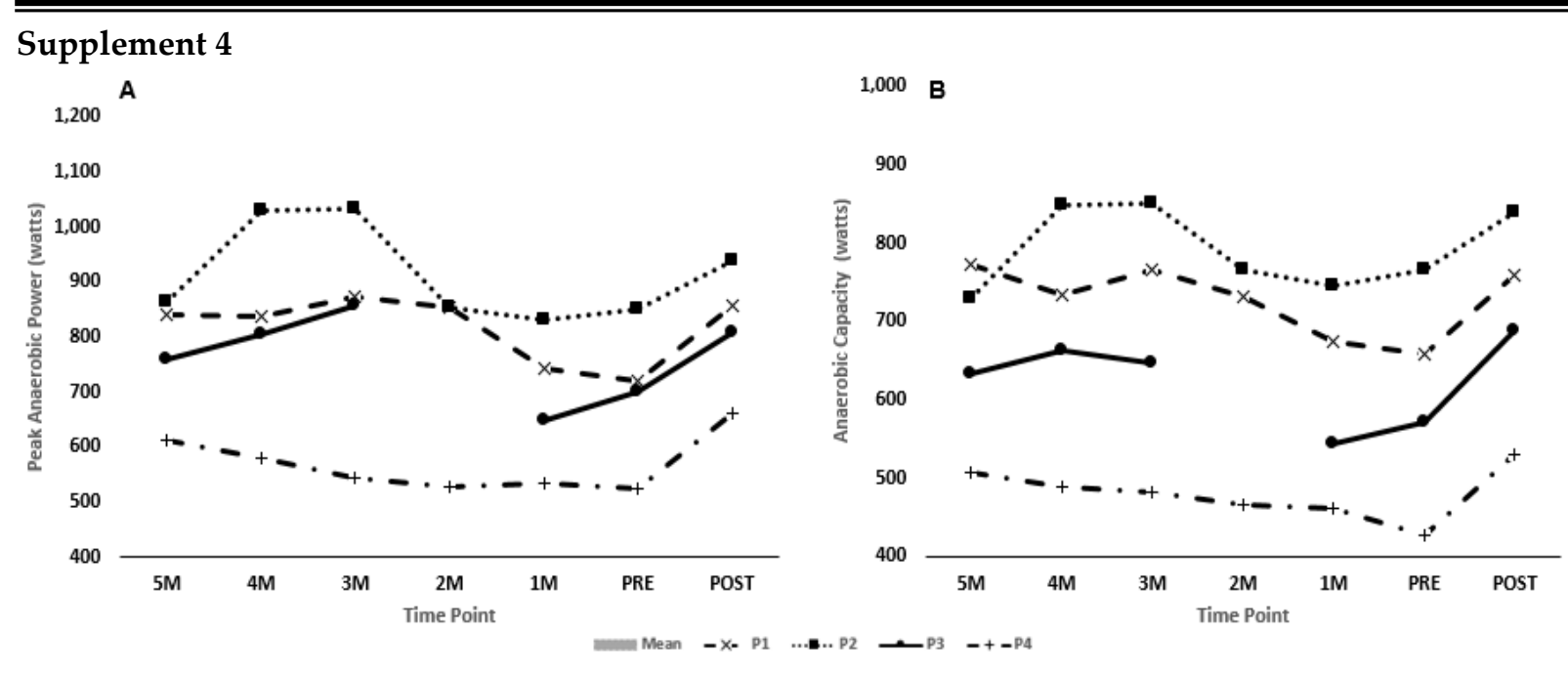

Supplement Figure (S4) A-B. Effects of Bodybuilding Competition Preparation on Anaerobic Power and Capacity. A Peak Anaerobic Power (watts), B Anaerobic Capacity (watts). Abbreviations. M months from final competition, PRE-final visit to the lab prior to last competition of the season, POST 8 weeks post the PRE laboratory visit, LBM lean body mass, P1, P2, P3, P4 participant 1, 2, 3, 4.

\section{Supplement 5}

Participant Number 4 - Pre-Competition Progress Photographs

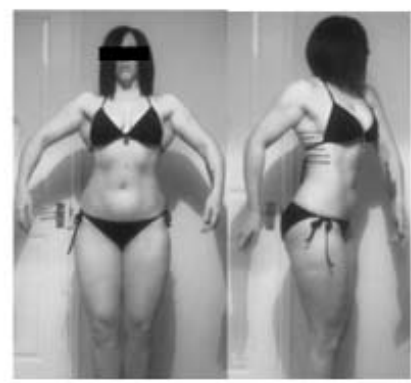

Starting Point -7 Months Pre

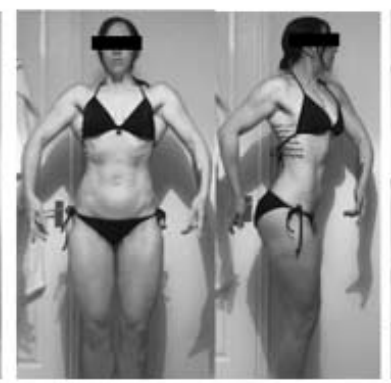

3 Months Pre

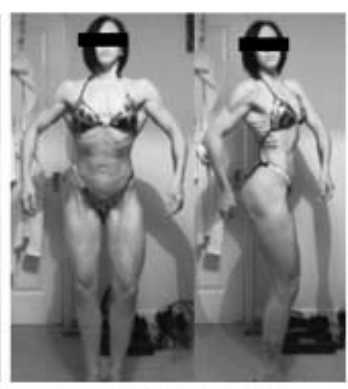

1 Month Pre

Participant Number 4 - Competition \& Post Competition Photographs

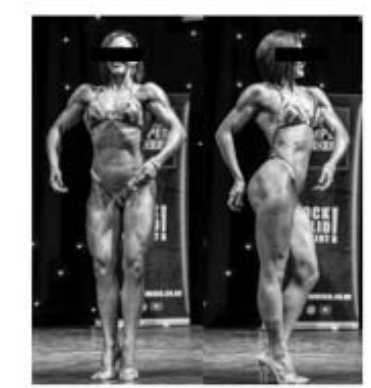

Competition Day - 7 Months Dieting

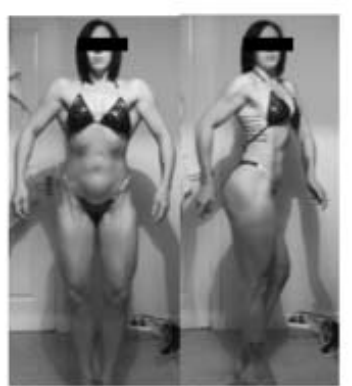

1 Month Post Competition Day 


\section{References}

Andersen RE, Barlett SJ, Morgan GD, Brownell KD. Weight loss, psychological, and nutritional patterns in competitive male body builders. Int J Eat Disord, 1995; 18: 49-57

Andersen RE, Brownell KD, Morgan GD, Bartlett SJ. Weight loss, psychological, and nutritional patterns in competitive female bodybuilders. Eat Disord, 1998: 6; 2: 159-167

Alwan N, Moss SL, Elliot-Sale KJ, Davies IG, Enright K. A Narrative Review on Female Physique Athletes: The Physiological and Psychological Implications of Weight Management Practices. Int J Sport Nutr Exerc Metab, 2019; 1-8

Attia A, Hachana Y, Chaabène H, Gaddour A, Neji Z, Shephard RJ, Chelly MS. Reliability and validity of a 20-s alternative to the Wingate anaerobic test in team sport male athletes. PloS One, 2014; 9: e114444

Baird MF, Graham SM, Baker JS, Bickerstaff GF. Creatine-kinase-and exercise-related muscle damage implications for muscle performance and recovery. J Nutr Metab, 2012; 2012: 960363

Chappell AJ, Simper TN. Nutritional peak week and competition day strategies of competitive natural bodybuilders. Sports, 2018; 6: 126

Compher C, Frankenfield D, Keim N, Roth-Yousey L. Evidence Analysis Working Group. Best practice methods to apply to measurement of resting metabolic rate in adults: a systematic review. J Am Diet Assoc, 2006; 106: 881 - 903

Cunningham JJ. A Reanalysis of the factors influencing basal metabolic rate in normal adults. Am J Clin Nutr, 1980; 33: 2372 - 2374

Cuzzolaro M, Vetrone G, Marano G, Garfinkel PE. The Body Uneasiness Test (BUT): development and validation of a new body image assessment scale. Eat Weight Disord, 2006; 11: 1-3

Devrim A, Bilgic P, Hongu N. Is there any relationship between body image perception, eating disorders, and muscle dysmorphic disorders in male bodybuilders? Am J Mens Health, 2018; 12: 1746-1758

Dorgan JF, Judd JT, Longcope C, Brown C, Schatzkin A, Clevidence BA, Campbell WS, Nair PP, Franz C, Kahle L, Taylor PR. Effects of dietary fat and fiber on plasma and urine androgens and estrogens in men: a controlled feeding study. Am J Clin Nutr, 1996; 64: 850-855

Fagerberg P. Negative consequences of low energy availability in natural male bodybuilding: A review. Int $J$ Sport Nutr Exerc Metab, 2018; 28: 385-402

Garner DM, Garfinkel PE. The Eating Attitudes Test: An index of the symptoms of anorexia nervosa. Psychol Med, 1979; 9: 273-279

Hämäläinen E, Adlercreutz H, Puska P, Pietinen P. Diet and serum sex hormones in healthy men. J Steroid Biochem, 1984; 20: 459-464

Helms ER, Fitschen PJ, Aragon AA, Cronin J. Schoenfeld BJ. Recommendations for natural bodybuilding contest preparation: resistance and cardiovascular training. J Sports Med Phys Fitness, 2015; 55: 164-178

Helms ER, Prnjak K, Linardon J. Towards a sustainable nutrition paradigm in physique sport: a narrative review. Sports, 2019; 7: 172

Helms ER, Aragon AA, Fitschen PJ. Evidence-based recommendations for natural bodybuilding contest preparation: nutrition and supplementation. JISSN, 2014; 11: 20

Heymsfield SB, Gonzalez MC, Shen W, Redman L, Thomas D. Weight loss composition is one-fourth fat-free mass: a critical review and critique of this widely cited rule. Obes Rev, 2014; 15: 310-321

Hulmi JJ, Isola V, Suonpää M, Järvinen NJ, Kokkonen M, Wennerström A, Nyman K, Perola M, Ahtiainen JP, Häkkinen $\mathrm{K}$. The effects of intensive weight reduction on body composition and serum hormones in female fitness competitors. Front Physiol, 2017; 7: 689

Jackson AS, Pollock ML. Generalized equations for predicting body density of men. Br J Nutr, 1978; 40: 497504

Mäestu J, Eliakim A, Jürimäe J, Valter I, Jürimäe T. Anabolic and catabolic hormones and energy balance of the male bodybuilders during the preparation for the competition. J Strength Cond Res, 2010; 24:10741081

Mitchell L, Slater G, Hackett D, Johnson N, O'connor H. Physiological implications of preparing for a natural male bodybuilding competition. Eur J Sport Sci, 2018;18: 619-629 
Mitchell L, Murray SB, Hoon M, Hackett D, Prvan T, O'Connor H. Correlates of muscle dysmorphia symptomatology in natural bodybuilders: Distinguishing factors in the pursuit of hyper-muscularity. Body Image, 2017a; 22: 1-5

Mitchell L, Murray SB, Cobley S, Hackett D, Gifford J, Capling L, O'Connor H. Muscle dysmorphia symptomatology and associated psychological features in bodybuilders and non-bodybuilder resistance trainers: A systematic review and meta-analysis. Sports Med, 2017b; 47: 233-259

Müller W, Lohman TG, Stewart AD, Maughan RJ, Meyer NL, Sardinha LB, Kirihennedige N, Reguant-Closa A, Risoul-Salas V, Sundgot-Borgen J, Ahammer H. Subcutaneous fat patterning in athletes: selection of appropriate sites and standardisation of a novel ultrasound measurement technique: ad hoc working group on body composition, health and performance, under the auspices of the IOC Medical Commission. Br J Sports Med, 2016; 50: 45-54

Petrizzo J, DiMenna FJ, Martins K, Wygand J, Otto RM. Case Study: The Effect of 32 Weeks of Figure-Contest Preparation on a Self-Proclaimed Drug-Free Female's Lean Body and Bone Mass. Int J Sport Nutr Exe, 2017; 27: 543-549

Robinson SL, Lambeth-Mansell A, Gillibrand G, Smith-Ryan A, Bannock L. A nutrition and conditioning intervention for natural bodybuilding contest preparation: case study. JISSN, 2015; 12: 20

Rohrig BJ, Pettitt RW, Pettitt CD, Kanzenbach TL. Psychophysiological tracking of a female physique competitor through competition preparation. Int J Exerc Sci, 2017; 10: 301-311

Rossow LM, Fukuda DH, Fahs CA, Loenneke JP Stout JR. Natural bodybuilding competition preparation and recovery: a 12-month case study. Int J Sport Phys and Perform, 2013; 8: 582-592

Schofield WN. Predicting basal metabolic rate, new standards and review of previous work. Hum Nutr Clin Nutr, 1985; 39: 5 - 41

Shim JS, Oh K, Kim HC. Dietary assessment methods in epidemiologic studies. Epidemiol health, 2014; 36: e2014009

Speakman JR, Selman C. Physical activity and resting metabolic rate. Proc Nutr Soc, 2003; 62: 621 - 634

Spendlove J, Mitchell L, Gifford J, Hackett D, Slater G, Cobley S, O'Connor H. Dietary intake of competitive bodybuilders. Sports Med, 2015; 45:1041-1063

Spielberger CD, Gorsuch RL. State-Trait Anxiety Inventory for Adults. Manual, Instrument and Scoring Guide. Mind Garden Inc, 1983

Tinsley GM, Trexler ET, Smith-Ryan AE, Paoli A, Graybeal AJ, Campbell BI, Schoenfeld BJ. Changes in Body Composition and Neuromuscular Performance Through Preparation, 2 Competitions, and a Recovery Period in an Experienced Female Physique Athlete. J Strength Cond Res, 2018; 33: 1823 - 1939

Trexler ET, Hirsch KR, Campbell BI, Smith-Ryan AE. Physiological changes following competition in male and female physique athletes: a pilot study. Int J Sport Nutr Exerc Metab, 2017; 27: 458-466

\section{Corresponding author:}

\section{Andrew. J. Chappell}

School of Pharmacy and Life Science, Robert Gordon University,

Garthdee Road, Aberdeen, AB10 7AQ, United Kingdom

E-mail: Andrew.chappell.ac@gmail.com 